\title{
Calculation of Spur Gear Dynamic Transmission Error in Consideration of the Progressive Engagement of Compliant Profile-modified Teeth
}

\author{
DIMITRIOU Konstantinos ${ }^{1}$, and FOKAS Nikolaos ${ }^{1, \text { a }}$ \\ ${ }^{1}$ Psyche engineering systems and technologies BV, Koningin Juliana Plein 10, 2595 AA Den Haag, Netherlands
}

\begin{abstract}
A simple yet accurate model is developed for the dynamical simulation of profile-modified gears, considering the effects of progressive tooth engagement, stiffness, elastohydrodynamic lubricant film formation and hysteresis. The real path of contact, stiffness and elastohydrodynamic lubricant film thickness are calculated for various operating conditions and the results are input to the dynamical simulation, resulting in a prediction of the dynamic transmission error.
\end{abstract}

\section{Introduction}

Perfectly nominal tooth forms are not ideal for gear operation, as the dynamic deformation of the loaded gear teeth leads to impact in the beginning of tooth engagement [1]. This is particularly undesirable, as it can lead to significant vibration and noise, especially when the transmitted loads (and corresponding tooth deflections) are high. The most common remedy is to calculate the deflection of a pair of teeth at the beginning of their mesh cycle for the nominal operating load and then to modify their profiles by a corresponding amount, so as to induce progressive tooth engagement. Almost all of the research conducted on gear dynamic behavior is limited to unmodified conjugate gears [2-8], thereby leaving these questions regarding the dynamic behavior of gears with profile modifications unanswered. It used to be that profile modifications were mostly considered at the level of static and/ or kinematic calculations [9-10]; however this trend is changing [11-12].

To model the effect of progressive tooth engagement in gears with profile modifications, tooth geometry and engagement progression are examined using an advanced non-conjugate tooth contact theory, which introduces a gear-specific form for the parametrical equations of tooth profile tangency to achieve the explicit solution of these equations. After ascertaining the kinematical characteristics of the modified gears, dynamic simulation of these gears has been conducted over a wide range of speeds using a combined geometric-kinematic-dynamic model based on known analytical and numerical models for calculating gear geometry, contact and kinematics [13-18] and mechanical response of the elastic tooth mesh, also in consideration of modified tooth geometries and elastohydrodynamic lubrication-induced tooth surface deformations [19-30].

\section{Kinematical Modelling}

Consider a pair of spur gears 1 and 2 having points $O_{1}$ and $\mathrm{O}_{2}$ as their respective centers of rotation and continuous planar curves $c_{1}$ and $c_{2}$, such that they represent the working flanks of two teeth belonging to gears 1 and 2 respectively. To distinguish between different teeth, teeth are identified by integers $O_{1}$ and $o_{2}$ so that, for example, tooth $o_{1}=5$ is the fifth tooth of gear 1. In the general case of meshing of two non-conjugate profiles the operating pitch circle of gear 2 can be considered to slip relatively to that of gear 1 (reference gear) by a slip angle $\vartheta_{s}$ (transmission error) and the angular positions of the tooth profiles are given by the following equations:

$$
\begin{aligned}
& \vartheta_{1,01}=\vartheta_{1}+2 \pi \frac{o_{1}-1}{z_{1}} \\
& \vartheta_{2,02}+\vartheta_{s}=\vartheta_{2}+2 \pi \frac{o_{2}-1}{z_{2}}+\vartheta_{s}
\end{aligned}
$$

where $\vartheta_{2}$ is the theoretical angular position of gear 2 for the case of zero slip between the operating pitch circles (case of uniform motion transmission), $\vartheta_{2,02}$ is the corresponding theoretical angular position of the working profile of tooth $\mathrm{O}_{2}$ and $\mathrm{z}$ is the number of teeth. This translates into the following relationship:

$$
d \vartheta_{1}=-i_{12} d \vartheta_{2}
$$

where $i_{12}$ is the transmission ratio, and finally because of Eqs. (1) and (2):

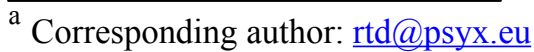




$$
\frac{d \vartheta_{2,02}}{d \vartheta_{1,01}}=\frac{d \vartheta_{2}}{d \vartheta_{1}}=-\frac{1}{i_{12}}
$$

If at angular positions $\vartheta_{1,01}=\vartheta_{2,02}=0$ curves $c_{1}$ and $c_{2}$ are described by the vector functions:

$$
\begin{aligned}
& \mathbf{r}_{1}=\mathbf{f}_{1}\left(r_{1}\right) \\
& \mathbf{r}_{2}=\mathbf{f}_{2}\left(r_{2}\right)
\end{aligned}
$$

where $r_{1}, r_{2}$ are the corresponding radii from the centers $O_{1}$ and $\mathrm{O}_{2}$ (also corresponding to the position vectors marked in boldface), the incorporation of the slip angle yields from Eqs. (5), (6) the following equations:

$$
\begin{aligned}
& \mathbf{r}_{1}=\mathbf{R}_{1} \mathbf{f}_{1}\left(r_{1}\right) \\
& \mathbf{r}_{2}=\mathbf{R}_{2} \mathbf{R}_{s} \mathbf{f}_{2}\left(r_{2}\right)
\end{aligned}
$$

where $\quad \mathbf{R}_{1}=\mathbf{R}_{1}\left(\vartheta_{1,01}\right) \quad, \quad \mathbf{R}_{2}=\mathbf{R}_{2}\left(\vartheta_{2,02}\right) \quad$ and $\mathbf{R}_{s}=\mathbf{R}_{s}\left(\vartheta_{s}\right)$ are the rotary translation matrices corresponding to planar rotation by the corresponding angles $\vartheta_{1,01}, \vartheta_{2,02}$ and $\vartheta_{s}$. At the contact point of the mating flanks the requirement for continuity imposes the following contact conditions:

$$
\begin{aligned}
& \mathbf{r}_{1}-\mathbf{r}_{2}=\overrightarrow{O_{1} O_{2}} \\
& \frac{d \mathbf{r}_{1}}{d r_{1}} \times \frac{d r_{2}}{d r_{2}}=\mathbf{0}
\end{aligned}
$$

By introducing Eqs. (7) and (8) into Eqs. (9) and (10), the general equations of meshing are obtained in the following form:

$$
\begin{aligned}
& \mathbf{R}_{1} \mathbf{f}_{1}-\mathbf{R}_{2} \mathbf{R}_{s} \mathbf{f}_{2}=\overrightarrow{O_{1} O_{2}} \\
& \mathbf{R}_{1} \mathbf{f}_{1}^{\prime} \times \mathbf{R}_{2} \mathbf{R}_{s} \mathbf{f}_{2}^{\prime}=\mathbf{0}
\end{aligned}
$$

where $r_{1}=r_{1}\left(\vartheta_{1,01}\right) \quad, \quad r_{2}=r_{2}\left(\vartheta_{2,02}\right) \quad \kappa \alpha l$ $\vartheta_{s}=\vartheta_{s}\left(\vartheta_{1,01}\right)$.

To solve these equations of meshing, we must determine the function $\vartheta_{s}\left(\vartheta_{1,01}\right)$ when functions $\mathbf{f}_{1}\left(r_{1}\right)$ and $\mathbf{f}_{2}\left(r_{2}\right)$ are given.

By solving Eq. (11) the following expression can be obtained:

$$
r_{2}=\left\|\mathbf{R}_{1} \mathbf{f}_{1}-\overrightarrow{O_{1} O_{2}}\right\|=U_{1}\left(\vartheta_{1,01}, r_{1}\right)
$$

In addition, the vectors $\mathbf{f}_{2}$ and $\mathbf{R}_{1} \mathbf{f}_{1}-\overrightarrow{O_{1} O_{2}}$ form an angle of $\vartheta_{2,02}+\vartheta_{s}$, hence:

$$
\vartheta_{2,02}+\vartheta_{s}= \pm \cos ^{-1}\left\{\frac{\left[\mathbf{R}_{1} \mathbf{f}_{1}-\overrightarrow{O_{1} O_{2}}\right] \cdot \mathbf{f}_{2}}{\left\|\mathbf{R}_{1} \mathbf{f}_{1}-\overrightarrow{O_{1} O_{2}}\right\|\left\|\mathbf{f}_{2}\right\|}\right\}
$$

and finally because of Eqs. (11) and (13) the following equation is true:

$\vartheta_{s}=-\vartheta_{2,02} \pm \cos ^{-1}\left\{\frac{1}{r_{2}^{2}}\left[\mathbf{R}_{1} \mathbf{f}_{1}-\overrightarrow{O_{1} O_{2}}\right] \cdot \mathbf{f}_{2}\right\}=U_{2}\left(\vartheta_{1,01}, r_{1}, r_{2}\right)$

Because vectors $\mathbf{f}_{1}^{\prime}$ and $\mathbf{f}_{2}^{\prime}$ are coplanar, Eq. (12) is equivalent to:

$$
\left[\mathbf{R}_{1} \mathbf{f}_{1}^{\prime} \times \mathbf{R}_{2} \mathbf{R}_{s} \mathbf{f}_{2}^{\prime}\right] \cdot \mathbf{z}=U_{3}\left(\vartheta_{1,01}, r_{1}, r_{2}, \vartheta_{s}\right)=0
$$

where $\mathrm{z}$ is the normal unitary vector to the common profile plane. From Eqs. (13), (15) and (16) the following equation is obtained:

$$
U_{3}\left(\vartheta_{1,01}, r_{1}, U_{1}\left(\vartheta_{1,01}, r_{1}\right), U_{2}\left(\vartheta_{1,01}, r_{1}, U_{1}\left(\vartheta_{1, o 1}, r_{1}\right)\right)\right)=0
$$

from which the value $r_{1}$ corresponding to a given position $\vartheta_{1,01}$ can be calculated. Following that, $r_{2}$ is extracted from Eq. (13) and $\vartheta_{s}$ is finally calculated from Eq. (15).

\section{Dynamical Modelling}

For the dynamical model we consider the effects of stiffness, tooth friction and hysteresis, which are implemented in the form of stiffness and friction force coefficients $\mathrm{K}$ and Fr, which are computed using FEA and elastohydrodynamic lubrication theory.

The model considers a single torsional degree of freedom and is as follows:

$$
\begin{aligned}
& J_{1} \dot{\boldsymbol{\omega}}_{1}+c_{1} \boldsymbol{\omega}_{1}=-\sum \overrightarrow{O_{1} A} \times\left(\mathbf{F}_{n}+\mathbf{F}_{s}\right)+\mathbf{T}_{1} \\
& J_{2} \dot{\boldsymbol{\omega}}_{2}+c_{2} \boldsymbol{\omega}_{2}=\sum \overrightarrow{O_{2} A} \times\left(\mathbf{F}_{n}+\mathbf{F}_{s}\right)+\mathbf{T}_{2}
\end{aligned}
$$

where $\mathrm{J}$ is the moment of inertia, $\omega$ is the angular velocity, $\mathrm{A}$ is the instantaneous mesh contact point, $\mathrm{O}$ is the gear centre of rotation, $F_{n}$ is the mesh normal force, $F_{s}$ is the mesh friction force and $\mathrm{T}$ is the external torque acting on the gear shaft. By introducing the stiffness and friction force coefficients the dynamic equations become:

$$
\begin{aligned}
& J_{1} \ddot{\vartheta}_{1}+c_{1} \dot{\vartheta}_{1}=\left[ \pm \frac{1}{i_{12}} K+F r_{1}\right] \vartheta_{s} \pm \frac{c_{\vartheta}}{i_{12}} \frac{d}{d t} \vartheta_{s}+T_{1} \\
& J_{2} \ddot{\vartheta}_{2}+c_{2} \dot{\vartheta}_{2}=\left[K+F r_{2}\right] \vartheta_{s}+c_{\vartheta} \frac{d}{d t} \vartheta_{s}+T_{2}
\end{aligned}
$$

\section{Results And Discussion}

The preceding analysis was used to model a $20^{\circ}$ involute standard spur gear pair with transmission ratio $21 / 50$ rotating at $100 \mathrm{rpm} / 400 \mathrm{Nm}$ (case a), $1000 \mathrm{rpm} / 40 \mathrm{Nm}$ (case b) and $5000 \mathrm{rpm} / 8 \mathrm{~N} / \mathrm{m}$ (case c), thus keeping the power constant. The tip of gear 2 was relieved starting $1.0 \mathrm{~mm}$ below the tip to achieve a tip thickness reduction 
of $0.1 \mathrm{~mm}$ (see Fig. 1). The calculated path of contact is shown in Fig. 2. The stiffness coefficient variation during a mesh cycle resulting from progressive tooth engagement due to the applied profile modifications is plotted in Fig. 2, top left. The sloped transitional regions as compared to the abrupt changes of the unmodified profiles testify to the effect of profile modifications on the progressive tooth engagement.

This gear configuration was simulated dynamically for the three different scenarios a-c, where the mean elastohydrodynamic lubricant film thickness was calculated using the theory by Dowson and Higginson [30] and shown in Fig. 2, bottom left. The calculated dynamic response curves are shown in Fig. 2, right. The curve corresponding to case c confirms the significance of the formation of an elastohydrodynamic lubricant film on gear transmission error; the driven gear actually begins to lead at high speeds as a result of the increased lubricant film thickness, which is translated into negative transmission error values.

\section{Conclusion}

The proposed model allowed the dynamical simulation of profile-modified gears, considering the effects of progressive tooth engagement, stiffness, elastohydrodynamic lubricant film formation and hysteresis. The model's predictions for the real path of contact, stiffness and elastohydrodynamic lubricant film thickness under for various operating conditions showed expected behaviours and the computed dynamic transmission error, especially in terms of its sensitivity to the operating speed, even leading to negative transmission error values, is highly plausible.

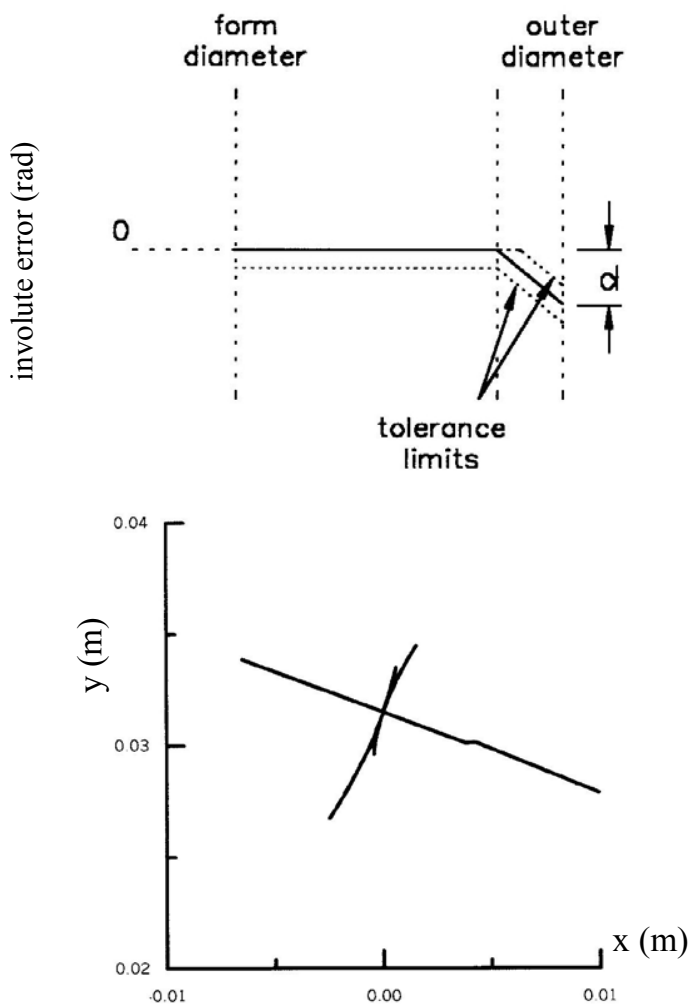

Figure 1. Tooth profile modification chart (left) and calculated path of contact (right)
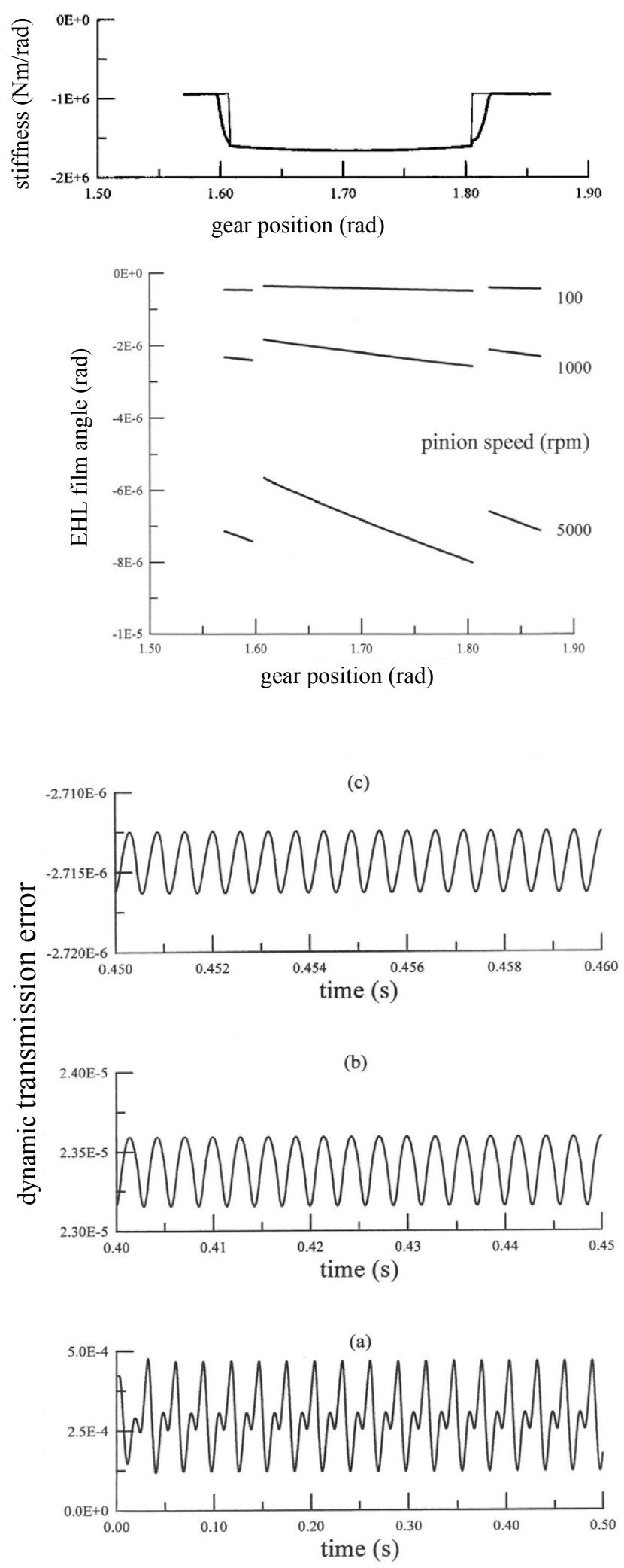

Figure 2. Calculated mesh stiffness coefficient (Nm/rad) for modified (thick line) and unmodified (thin line) teeth (top left), calculated EHL film angle (rad) for different pinion speeds (bottom left) vs position (rad) and simulated dynamic transmission error (rad) vs time (s) (right). 


\section{References}

1. Townsend D. P.: Dudley's Gears Handbook, McGraw-Hill, New York (1992)

2. Kasuba R., and Evans J. W.: Journal of Mechanical Design, Vol. 103(2) (1981), pp. 398-409

3. Cai Y.: Journal of Mechanical Design, Vol. 117(3) (1994), pp. 460-469

4. Velex P., and Maatar M.: Journal of Sound and Vibration, Vol. 191(5) (1996), pp. 629-660

5. Lin H.H., Huston R.L., and Coy J.J.: Journal of Mechanical Design, Vol. 110(2) (1988), pp. 221-225

6. Liou C. H., Lin H. H., Oswald F. B., and Townsend D. P.: Journal of Mechanical Design, Vol. 118(3) (1996), pp. 439-443

7. Yoon K.Y., and Rao S.S.: Journal of Mechanical Design, Vol. 118(1) (1996), pp. 1-6

8. Velex P., and Flamand L.: Journal of Mechanical Design, Vol. 118(1) (1996), pp. 7-14

9. Litvin F.L.: Gear Geometry and Applied Theory, Prentice Hall, NJ (1994)

10. Litvin F.L., Chen J.-S., Lu J., and Handschuh R.F.: Journal of Mechanical Design, Vol. 118(4) (1996), pp. 561-567

11. Spitas C., and Spitas V.: Journal of Multi-Body Dynamics, Vol. 220(4) (2006), pp. 273-282

12. Andersson A., Vedmar L.: Journal of Sound and Vibration, Vol. 260(2) (2003), pp. 195-212

13. Spitas C., and Spitas V.: Mechanism and Machine Theory, Vol. 46(7) (2011), pp. 869-879

14. Spitas C.: Journal of Sound and Vibration, Vol. 324(1-2) (2009), pp. 297-316

15. Spitas C., and Spitas V.: Applied Mathematical Modelling, Vol. 32(10) (2008), pp. 2162-2171

16. Litvin F.L., Gonzalez-Perez I., Fuentes A., Hayasaka K., and Yukishima K.: Mathematical and Computer Modelling, Vol. 42(9-10) (2005), pp. 1063-1078
17. Spitas C., Costopoulos T., and Spitas V.: Inverse Problems in Science and Engineering, Vol. 16(2) (2007), pp. 171-186

18. Spitas V., Costopoulos T., and Spitas C.: Mechanism and Machine Theory, Vol. 42(6) (2007), pp. 751-762

19. Litvin F.L., Fuentes A., Gonzalez-Perez I., Carnevali L., Kawasaki K., Handschuh R.F.: Computer Methods in Applied Mechanics and Engineering, Vol. 192 (2003), pp. 3619-3655

20. Spitas C., and Spitas V.: Journal of Mechanical Engineering Science, Vol. 220 (8) (2006), pp. 13051313

21. Arafa M.H., and Megahed M.M.: Journal of Mechanical Engineering Science, Vol. 213 (1999), pp. 569-579

22. Spitas C., Spitas V., Amani A., and Rajabalinejad M.: Acta Mechanica, Vol. 225(2) (2014), pp. 361-371

23. Chaari F., Takhfakh T., and Haddar M.: European Journal of Mechanics - A/Solids, Vol. 28(3) (2009), pp. $461-468$

24. Spitas V., and Spitas C.: Mechanics Based Design of Structures and Machines, Vol. 35(2) (2007), pp. 163178

25. Spitas C., and Spitas V.: Mechanics Based Design of Structures and Machines, Vol. 36(2) (2008), 189-211

26. Spitas V., and Spitas C.: Acta Mechanica, Vol. 193(1-2) (2007), pp. 113-126

27. Spitas C., and Spitas V.: Mechanics Based Design of Structures and Machines, Vol. 35(1) (2007), pp. 5973

28. Pimsarn M., Kazerounian K.: Mechanism and Machine Theory, Vol. 37(8) (2002), pp. 769-786

29. Spitas V., Spitas C.: Journal of Mechanical Engineering Science, Vol. 221 (2006), pp. 479-488

30. Dowson D., Higginson G.R.: Elastohydrodynamic Lubrication, Pergamon Press, Oxford (1966) 